et si nous portons sur la droite ainsi tracée une longueur OB telle que :

$$
\mathrm{OB}=\frac{\mathbf{e}_{\mathrm{clff}}}{\sqrt{\mathbf{R}^{2}+\mathbf{L}^{2} \boldsymbol{\omega}^{2}}}=\mathbf{i}_{1 \mathrm{ell}}
$$

nous aurons représenté en grandeur et en phase le courant $\mathbf{i}_{1}$.

Le courant $i_{2}$ étant en phase avec e, nous portons sur $\mathbf{O A}$ une longueur $\mathrm{OC}$ telle que :

$$
\mathrm{OC}=\frac{\mathbf{e}_{\mathrm{eff}}}{\boldsymbol{\rho}}=\mathbf{i}_{\mathrm{gel}}
$$

Enfin le courant $\mathbf{i}_{3}$ décalé à go en avant sur la force électromotrice sera représenté par $O D$ tel que :

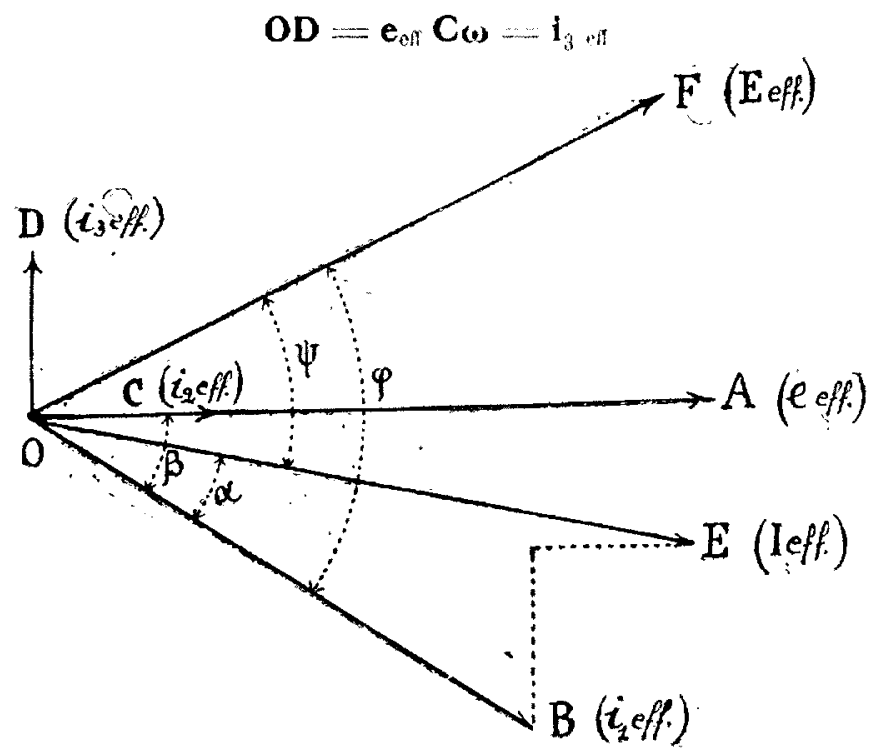

Fig. 4

Le courant de ligne I s'obtient on faisant la résultante géométrique de $\mathbf{i}_{1} \mathbf{i}_{2} \mathbf{i}_{3}$; on trouve ainsi $\mathbf{O E}$.

Si nous faisons en dessus de $\mathrm{OE}$ un angle $\mathrm{I}$ tel que :

$$
\operatorname{tg} \Psi=\frac{L^{\prime}(\omega)}{R^{\prime}}
$$

et si nous portons sur la droite ainsi considerée une longueur of telle que :

$$
\mathbf{O F}=\mathbf{O E} \sqrt{\mathbf{R}^{\mathbf{2}}+\mathbf{L}^{\mathbf{2}} \boldsymbol{\omega}^{\mathrm{g}}}
$$

nous aurons représenté en grandeur et en phase la force électromotrice $\mathbf{E}$ de l'alternateur. Mais cette longueur $\mathbf{O F}=\mathbf{E}$ est une des données du problème puisque l'alternateur est connu; il s'ensuit que l'échelle du dessin est fixée ct que la longueur $\mathbf{O A}$ dont nous sommes partis n'est plus arbitraire.

On voit enfin sur la figure que le décalage $\varphi$ cherché du courant $i_{1}$ sur la force électromotrice $\mathbf{E}$ est la somme des deux angles $\Psi^{*}$ et $x$.

$$
\varphi=\Psi+\alpha
$$

La construction précédente donne donc tous les éléments de la question et la discussion de l'épure donnerait les mêmes conclusions que la solution analytique.

\section{J. Courbier,}

Ancien ileve de l'Ecole Polytechnique at de l'Ecole Supérieure d'Electricite de Paris Licencié en Droit.
Réflexions sur les études préliminaires à l'aménagement des chutes d'eau

(Suite).

\section{II. - Avec quelle précision peut-on connaitre la puissance des chutes d'eau?}

\author{
10 Vue d'ensemble.
}

L'équation: $\quad \mathbf{Q H}=\mathbf{K}^{2}$

entre le débit, la hauteur de chute et la puissance nous domne entre les variations finies et absolues des paramètres qu'elle rapproche l'équation:

$$
د\left(\mathbf{K}^{2}\right)=\mathbf{H} . \Delta \mathbf{Q}+\mathbf{Q} \Delta \mathbf{H}
$$

et, entre les variations relatives des mêmes éléments,

$$
\frac{\Delta\left(\mathbf{K}^{2}\right)}{\mathbf{K}^{2}}=\frac{\Delta \mathbf{Q}}{\mathbf{Q}}+\frac{\Delta \mathbf{H}}{\mathbf{H}}
$$

équation qui lie, par conséquent, l'erreur relative àcraindre sur le résultat à celles qui affectent les mesures des éléments. Elle montre que, pour une erreur déterminée, pour un tant pour cent de perte préalablement consenti, l'erreur permise sur la hauteur devra être d'autant moindre que celle quion aura commise sur le jaugeage aura été plus grande, et inversement.

Or, des deux paramètres composants, débit et hauteur de chute, le plus difficile à évaluer est, de beaucoup, le débit ; il a donc le plus de chances de troubler les résultats, quoiqu'on fasse, aussi ne saurait-on s'entourer de trop de soins pour son appréciation.

\section{$2^{\circ}$ Précision du jaugeage.}

Sans refaire ici la théorie du jaugeage, analysons sommairement cependant de quels éléments se compose cette quantité.

Pour plus de simplicité, supposons que la section transversale du cours d'eau sur lequel nous spéculons soit rectangulaire : cette hypothése suffit à accuser les tendances des variations.

m étant le coefficient de contraction de la nappe d'écoulement, $\mathbf{1}$ le poids spécifique de l'eau, h l'épaisseur en mètres de la dite nappe, $\mathbf{L}$ la largeur en mètres du cours d'eau, $\mathbf{U}$ la vitesse moyenne d'écoulement en metres, nous savons que le débit en litres (ou en kilogrammes) est, dans le cas du régime permanent qu'on suppose toujours réalisé :

$$
Q=m h L U .1000
$$

Nous en tirons:

$$
\frac{\Delta \mathbf{Q}}{\mathbf{Q}}=\frac{\Delta \mathbf{h}}{\mathbf{h}}+\frac{\Delta \mathbf{L}}{\mathrm{L}}+\frac{\Delta \mathbf{U}}{\mathbf{U}}
$$

$3^{\circ}$ Vitesse déduite de la charge.

Des trois éléments, largeur du cours d'eau, épaisseur de la lame déversante et vitesse, c'est le dernier qui est le plus difficile à mesurer. La vitesse résulte, en effet, de la combinaison d'une mesure de longueur avec une mesure de temps. 
Il va de soique, si on peut éliminer la mesure du temps, le résultat gagnera en rigueur.

C'est le bénéfice qu'on retire de toutes les méthodes qui font dépendre la connaissance de la vitesse de la mesure de l'altitude de la surface libre du courant au-dessus d'un point bien défini de l'arête par dessus laquelle il se déverse. Les mesures des durées ayant été faites, une fois pour toutes, avec une rigueur extrême, par les expérimentateurs qui ont dressé des tables de correspondance des vitesses et des altitudes en question, il n'y a plus, dès lors, à les refaire. Tels sont les jaugeages par déversoirs, par vannes, séparés ou combinés entre eux. Les ingénicurs auront souvent arantage à employer ces méthodes en montagne, au moment des plus basses eaux.

Cette altitude, $\mathbf{h}$, qu'on nomme la charge, est liée avec l'épaisseur $\mathbf{h}$ de la lame déversante par une loi compliquée et inconnue. Dans la pratique on dresse des tables du rap. port $\frac{h^{\prime}}{\mathbf{h}}$ et, moyennant cet artifice, la vitesse $U$ est réductible it la forme:

$$
\mathbf{U}=\mathbf{m}^{\prime} V \overline{\mathbf{2 g h}}
$$

On en tire :

$$
\frac{\Delta U}{U}=\frac{1}{2} \frac{\perp h}{h}
$$

ce qui permet de ramener l'équation (5) a la forme

$$
\frac{\Delta Q}{Q}=\frac{3}{2} \frac{\Delta h}{h}+\frac{\Delta L}{L}
$$

qui conjugue ensemble deux paramètres, $h$ et $\mathbf{L}$, au lieu de trois.

Dans l'état actuel de nos moyens, il est possible de mesurer $\mathbf{L}$ avec une approximation de $\frac{\mathbf{2 5}}{\mathbf{1 0}^{5}}$ dans les cas les plus favorables ct de $\frac{\mathbf{9 7}}{\mathbf{1 0}^{\mathbf{5}}}$ dans les cas les moins propices.

Quant à $\mathbf{h}$ sa détermination pourra se faire, le plus souvent, au moyen du tube hydrostatique du capitaine Boileau (ouvrage déjà cité) qui permet de faire les lectures au moins au demi-centimètre près et même, dans le cas des installations commodes, au $1 / 4$ de centimètre. Les lectures qu'on peut faire à la pointe sont susceptibles d'une précision encore plus grande; elles ne seront que rarement industrielles, elles constituent plutôt des procédés de laboratoire (I).

En général les cours d'eau peu larges, dont le débit est faible, sont facilement accessibles et il n'est pas déraisonnable d'admettre qu'un opérateur soigneux pourra toujours s'installer, s'il à affaire à eux, de manière à évaluer $h$ à $\mathrm{J} / 4$ de centimètte près, toutes corrections faites, et cela, soit avec le tube, soit avec une simple règle graduée.

De même, les charges, dans les cours d'eau à plus gros débit, pourront être estimées à $\mathrm{s} / 2$ centimètre près; enfin, dans les grands cours d'eau, à gros débit, des limnimètres pourront, s'ils sont convenablement disposés, permettre d'éliminer de la mesure le résultat de l'agitation superfi-

(1) Voir Poncecer et Lesbros. - Expériences hydrauliques sur les lois de l'écoulement de l'eau. Paris, $1827-1832$, et Botr.eau, op. cit. cielle du courant et de conclure la charge à I centimètre près ( 1 ).

Quel est alors, pour ces conditions, le degré de précision qu'on peut atteindre dans l'évaluation du débit, principalement pour les petits cours d'eau?

\section{4o Jangeage par déversoir.}

Appliquons aux débits spécifiques la formule qui lie le débit à la charge sur l'arête du déversoir. Dans ce cas $\mathbf{L}=\mathbf{1}$ et $\mathbf{Q}=\mathbf{q}$. Il vient alors :

$$
q=m^{\prime} h / 2 g h
$$

qui, résolue par rapport à $\mathbf{h}$, donne .

$$
h=-\sqrt[3]{\frac{q^{2}}{2 g m^{\prime 3}}}
$$

N'ayant en vue ici qu'un aperçu général de la variation de $\mathbf{q}$ avec $\mathbf{h}$, nous prendrons pour $\mathbf{m}$ ' la valeur $\mathbf{0 , 4 2}$ moyenne de toutes celles qu'il peut prendre et dont la liste, dressée par le capitaine Boileau dans son ouvrage cité est reproduite dans plusieurs aides-mémoire, entre autres dans celui

\begin{tabular}{|c|c|c|}
\hline $\mathbf{q}$ & h & $\begin{array}{l}\text { Incertitude maxima due } \\
\text { a l'erreur sur m' }\end{array}$ \\
\hline$o^{m 3} 00 ?$ & $o^{\text {moot }}$ & $\pm 0,00006$ \\
\hline$\alpha^{\mathrm{m} 3}$ oro & $0 \mathrm{mo}_{0}$ & $\pm 0,00030$ \\
\hline Om? 100 & $0^{m}+4^{2}$ & \pm 0.00102 \\
\hline $\mathrm{o}^{\mathrm{m} 3} 250$ & $0^{m} 262$ & $\pm \quad 0,00262$ \\
\hline $\mathrm{o}^{\mathrm{m} 3} 500$ & $\mathrm{om}_{4} \mathrm{I}_{6}$ & $\pm 0,00416$ \\
\hline $0^{m 3} 750$ & $0^{1115} 545$ & $\pm 0,00545$ \\
\hline
\end{tabular}
de Claudel. En agissant ainsi, nous n'altérons pas le lésultat de $1 / 100^{\circ}$ de sa valeur.

Nous pourrons alors dresser le petit tableau suivant :

$$
\text { TABLEAU I }
$$

Les trois premières valeurs de $\mathbf{h}$ peuvent être très aisément évaluées à $\mathrm{o}^{\mathrm{m}} \mathrm{O02} 5$ près ( $\mathrm{I} / 4$ de centimètre); on voit que l'erreur due à l'emploi d'une valeur approchée pour $\mathbf{m}$ ' est inférieure à l'erreur que peut apporter l'observation.

Les trois dernières valeurs de $\mathbf{h}$ peuvent ĉtre très aisément évaluées à $0^{\mathrm{m}} 005$ près ( $1 / 2$ centimètre), il apparaît aussi, dans ce cas, que l'erreur du calcul est inférieure i l'erreur due à l'observation, sauf pour la derniere, sans que, cependant, la perturbation soit bien grande.

Nous admettrons donc comme bonnes les valeurs de $\mathbf{h}$ calculées avec $\mathbf{m}^{\prime}=\mathbf{0 , 4 2}$; admettant $\Delta \mathbf{h}=\mathbf{0} 0025$ pour les trois premieres et $\Delta \mathbf{h}=\mathbf{0 m 0 0 5}$ pour les trois autres,

(1) En cela il ne peut être question que de limnimètres rudimentaires ne rappelant que de loin les marégraphes, médimarémètres, ete., instruments de précision, coûteux, stables et qui sont plutôt de véritables observatoires que des instruments utilisables pour l'industrie. Les limnimètres industriels permettent de lire les mouillages à a centimètre près. 
nous avons pour les débits spécifiques et les erreurs dont la mesure de la charge peut les affecter, le tahleau suivant:

TABIFA: II

\begin{tabular}{|c|c|c|c|}
\hline$q$ & $\frac{3}{2} \frac{\Delta h}{h}=\frac{1,5 \times 0^{n_{0}} 0025}{h}$ & $\mathbf{q}$ & $\frac{3}{2} \cdot \frac{\Delta h}{h}=\frac{\mathrm{T}, 3 \mathrm{X}}{\mathrm{h}}$ \\
\hline $\mathrm{O}^{\mathrm{m} 3} \mathrm{OOI}$ & 0,625 & $\mathrm{O}^{\mathrm{m} m} \cdot 250$ & 0,028626 \\
\hline $\mathrm{O}_{\mathrm{m}} 3010$ & 0,325 & $0^{\mathrm{m} 3}: 00$ & 0,018028 \\
\hline $0^{11+3} 100$ & 0,02648 & $0^{m ?} 7$ o & 0,013261 \\
\hline
\end{tabular}

Si nous joignons a ces valeurs celle de l'erreur relative possible sur l'évaluation de la largeur du cours d'eau $\left(\frac{97}{10^{5}}\right.$ quelle que soit cette largeur $)$ nous avons :

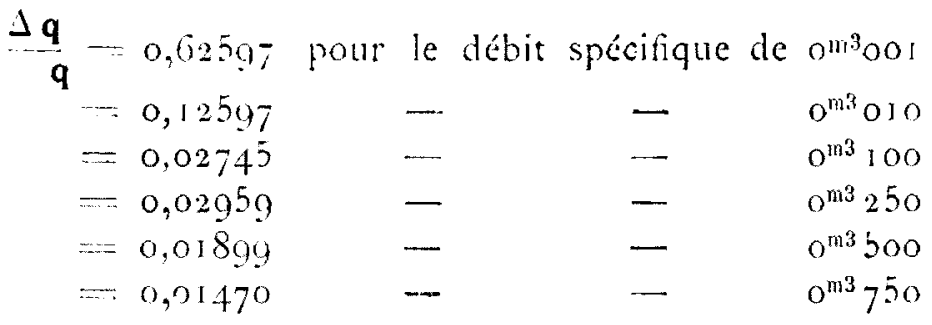

jo Jangeage par orifices.

Si, au lieu de jauger notre cours d'eau par le moyen des déversoirs, nous utilisions l'écoulement a travers des orifices en minces parois, nous trouverions pour charges sur l'arête supérieure d'un orifice de $\mathrm{o}^{\mathrm{m}} 2 \mathrm{O}$ de haut et de I mètre de linge:

\section{Tabifad III}

\begin{tabular}{|c|c|c|c|}
\hline$q$ & $\mathbf{h}$ & $\mathbf{q}$ & $\mathbf{h}$ \\
\hline $\mathrm{O}^{\mathrm{m} 3} \mathrm{OO} \mathrm{I}$ & $0^{1 n} 005$ & $O^{\mathrm{m} 3} 250$ & $\mathrm{O}^{1+2} 2 \mathrm{i}$ \\
\hline $0^{\mathrm{m} 3} \mathrm{O} \times 10$ & $0^{m_{0}} 024$ & $0^{\mathrm{m} 3} 500$ & $o^{m} 305$ \\
\hline $0^{1 \mathrm{~m} 3} 100$ & $o^{m}{ }_{1}{ }_{2}$ & $0^{\mathrm{m} 3}-50$ & $0^{m} 431$ \\
\hline
\end{tabular}

Ces charges sont un peu plus faibles que celles que demande le déversoir à égalité de débit.

D'autre part, les moyens de les mesurer sont ou des tubes ou des règles, comme dans le cas du déversoir, et les mesures seront entachées des mêmes inexactitudes. L'influence de celles-ci sera donc plus grande que dans le cas du jaugeage par déversoir. On ne devra done employer la méthode des orifices que lorsqu'on y trouvera, par ailleurs, des avantages réels. On comprend aussi pourquoi MM. Poncelet et Lesbros ont poussé la rigueur jusqu'à munir de verniers au $1: 10$ les règles qui leur accusaient l'amplitude des mouvements de leurs vannes.

\section{6o Résultats immédiats.}

Cette rapide analyse montre combien est délicate l'opération du jaugeage dans le cas des débits spécifiques faibles. Inversement, plus le débit spécifique est abondant, plus la mesure est facile. Mais, dans la pratique, il faut encore compter avec les difficultés matérielles de l'installation; or elles croissent vite avec le volume des eaux roulées par un cours d'eau et l'incommodité de l'installation vient tempérer, quelquefois, dans une très forte mesure, l'optimisme auquel on pourrait être porté à céder par suite de la réflexion précédente. (I)

Il faut aussi remarquer que les formules de jeaugeage données par les auteurs, Poncelet, Lesbros, Boileau, Morin, etc, ne sont qu'approchées et qu'elles ne rendent

(1) Cette propriété est génécrale. A près ce que nous venons de voir, la vérité de cette assertion semble intuitive: il ne sera peut-être pas hors de propos, toutefois, de l'échirer encore par d'autres exemples.

Dans certains cas favorables il est possible d'assimiler les cours d'eau à des canaux de faible pente et à berges régulières. Il existe, pour l'évaluation du débit de tels canaux, une assez grande variété de tormules. Si, dans le cas d'une peute définie, $\frac{1}{\mathbf{1 0 0 0}}$ par exemple, on les applique aux débits spécifiques pris comme type par nous, on pourra des débits remonter aux mokillages. Les chiffres que l'on trouve sont naturellement variables avec les formules, mais, dans le cas de la verticalité des rives, et toutes réserves faites sur le poids à accorder à chacun de ses procédés de calcul, ils ne semblent pas devoir s'écarter sensiblement des valeurs suivantes:

\begin{tabular}{|c|c|c|c|}
\hline $\mathbf{q}$ & $\mathbf{h}$ & $\mathbf{q}$ & $\mathbf{h}$ \\
\hline $0^{m i x}$ oor & omoos & $\left(3 m^{x} 250\right.$ & om 206 \\
\hline $0^{m} 3^{3} 010$ & $0^{\mathrm{m}} 024$ & $0^{14^{3} 500}$ & om 314 \\
\hline $0^{m^{3}} 100$ & on 086 & $o^{n^{3}} ; 50$ & $o^{m} 479$ \\
\hline
\end{tabular}

Tels qu'ils sont ils confirment toutefois notre vue sur la facilité du jaugeage des gros débits, en même temps qu'ils nous renseignent sur l'allure de la variation du mouillage avec le débit.

L.cs experiences directes, assez difficiles a installer du reste, permettraient seules de compléter les travaux des Prony, Darcy, Bazin, etc, de façon it corriger les résultats déduits des formules, en vue de serrer plus étroitement la généralité et la réalité des faits.

Quand les berges des cours d'eau sont irrégulières, ainsi que leur thalweg, on ne peut les assimiler à des canaux; si en outre leurs dimensions sont telles qu'on ne puisse les jauger par orifices ou déversoirs, it fautrecourir à d'autres procédés. On fait alors intervenir des mesures de vitesses prises au thalweg, conjointement avec des hypothèses sur la répartition des vitesses qui, combinées avec un nombre restreint de mesures opportunes, aboutissent à une expression empirique du débit en fonction du mouillage. Ce mouillage $\mathbf{h}$, est accusé sur un limnimètre et la formule donne le débit par le calcul d'un polynôme ordonné suivant les puissances, de $\mathbf{h}$. Admettons qu'on se soit arrêté à la $2^{e}$ puissance de $h$, on écrirait:

$$
\mathbf{q}=\mathbf{A} \mathbf{h}^{2}+\mathbf{B h}+\mathbf{c}
$$

A, B, c, étant des coefficients numériques propres au cours d'eau en expérience.

Il en résultera:

$$
\frac{\Delta \mathbf{q}}{\mathbf{q}}=\frac{\mathbf{2} \mathbf{A} \mathbf{h}}{\mathbf{A} \mathbf{h}^{2}+\mathbf{B h}+\mathbf{c}} \Delta \mathbf{h}-\frac{\mathbf{B}}{\mathbf{A h}^{2}-\mathbf{B h}+\mathbf{c}} \Delta \mathbf{h}
$$

Le ze terme du second membre décroit évidemment quand h croit; quant au premier il peut s'écrire, par suite de la division effectuée:

$\frac{2 \mathbf{A h}}{\mathbf{A} \mathbf{h}^{2}+\mathbf{B} \mathbf{h}+\mathbf{c}} \Delta \mathbf{h}=\Delta \mathbf{h}\left[\frac{\mathbf{2}}{\mathbf{h}}-\frac{\mathbf{B}}{\mathbf{A}} \cdot \frac{\mathbf{2}}{\mathbf{h}^{2}}+\frac{\stackrel{2}{\mathbf{h}}\left(\frac{\mathbf{B}^{2}}{\mathbf{A}}-\mathbf{c}\right)+\frac{\mathbf{2}}{\mathbf{h}^{2}} \cdot \frac{\mathbf{B c}}{\mathbf{A}}}{\mathbf{A \mathbf { h } ^ { 2 }}+\mathbf{B h}-\mathbf{c}}\right]$

forme sous laquelle il apparait clairement que la limite du quotient est $\mathbf{0}$, aussi bien que celle du reste, que, par conséquent, plus $\mathbf{h}$ est grand, plus est faible le multiplicateur de l'crreur commise sur la mesure de $\mathbf{h}$, moindre est donc son influence.

A fortiori ces conclusions seraient-elles exactes si on faisait entrer des puissances supérieures à $\mathbf{h}^{2}$ dans l'expression de $\mathbf{q}$ en fonction de $\mathbf{h}$; on sent que, dans de telles conditions la mesure de $\mathbf{h}$ au centimètre près donne des résultats très satisfaisants, toutes réserves faites sur l'exactitude des formules. 
compte de la réalité des faits que par a peu près. MM. Poncelet et Lesbros ont pu répondre de l'exactitude de certains de leurs résultats à $\frac{1}{200}$ près, mais, avec l'emploi des soins les plus méticuleux; quand ces précautions n'étaient pas de mise, ils ne répondaient pas de plus du $\frac{1}{\mathbf{5 0}}$. C'est aussi l'approximation que le capitaine Boileau s'estatt aché à obtenir dans la plupart de ses expériences. Il la considérait comme celle qui doit contenter l'industriel. Nous pensons que la suite de cette étude suggèrera à ceux qui auront bien voulu nous lire la pensée qu'on peut, dès maintenant, entrevoir des temps nouveaux où on demandera plus d'exactitude aux ingénieurs.

En tout cas, actuellement, il nous faut, de ce fait, augmenter de 0,02 les valeurs de $\frac{\Delta \mathbf{q}}{\mathbf{q}}$ que nous avons inscrites au tableau II et écrire (I) :

$$
\frac{\Delta \mathbf{Q}}{\mathbf{Q}}=0,02+\frac{\Delta q}{q}
$$

D'autre part, une erreur de plus de 12,5 "1" sur le débit, comme celle qu'accuse le tableau II pour un débit spécifique de ro litres (nous laissons de côté à dessein le débit de I litre, avec ses $62 \%$ il constitue un a fortiori), n'est pas tolérable et nous voyons que, dans le cas des débits très faibles, il faudra s'ingénier à l'amploi de tous les procédés possibles pour apprécier la charge, ou le débit, avec une rigueur bien autre que celle que nous avons admise. En mesurant, dans ces cas, $\mathbf{h}$ avec une règle à pointes et en évaluant cette mesure au millimètre près, on commettrait encore une erreur de 5 o . La suite montrera qu'il y a intérêt à la réduire et que, par conséquent, on peut, dans ces cas, s'essayer à obtenir l'approximation du demi-millimètre. C'est tres difficile.

(1) Les auteurs que nous venons de citer et, parmi eux le capitaine Boileau tout particulièrement, font observer que cette erreur $r=\frac{1}{50}$ sur le résultat de la formule, se produit parfois en plus, partois en moins, et avec une valeur qui n'est pas toujours $\frac{1}{50}$; on pourrait, s'appuyant sur le fait, être tenté de la considérer comme une erreur accidentelle et alors conclure que le theoreme de la probabilité composée (application du principe de Moivre) est applicable dizns le cás, ce qui donnerait

$$
\frac{\Delta \mathbf{Q}}{\mathbf{Q}}=\sqrt{\boldsymbol{x}^{2}+\left(\frac{\Delta \mathbf{q}}{\mathbf{q}}\right)^{\mathbf{2}}}
$$

On se tromperait, car la variation de $r_{\text {p }}$ peur être rattachée à des marches systématiques que les tableaux de Boiloau font très claire. ment ressortir. Ces marches sont compliquées, mais leur allure systématique est indéniable et, par dessus tout, la tendance à la compensation des valeurs de $r_{1}$ entre elles ne s'accuse pas nettement. Or, il ne faut pas perdre de vue que c'est là le caractère des écarts accidentels; tous ceux qui ne la présentent prs peuvent être systématisés et doivent être traités comme écarts systématiques. Par conséquent, la forme de l'erreur totale à craindre sur l'expression du jaugeage est bien :

$$
\frac{\Delta \mathbf{Q}}{\mathbf{Q}}=\boldsymbol{\eta}+\frac{\Delta \mathbf{q}}{\mathbf{q}}
$$

Plus la science progressera, plus se restreindra le domaine des écarts accidentels et plus s'étendra celui des écarts systématiques. C'est ainsi que l'homme arrive à la connaissance des Lois experimentales.
Les ingénieurs devront done renchérir sur la précision de de leurs instruments et de leurs procédés. En particulier, toutes les fois qu'ils le pourront,ils devront multiplier, répéter leurs mesures. Le calcul des probabilités leur apprend, en effet, que si sur une mesure isolée ils peuvent craindre une erreur accidentelle, $\boldsymbol{J} \mathbf{h}$, en refaisant $\mathbf{n}$ fois cette mesure et en supposant que chacune des opérations a été traitée avec le même soin, ils auront, avec la moyenne arithmétique de ces $\mathbf{n}$ mesures, une valeur qui ne s'écartera plus que de $\frac{\Delta \mathbf{h}}{V \mathbf{n}}$ de la valeur vraie. Malheureusement on est vite arrêté dans cette voie par la nature des choses et on ne peut jamais faire $\mathbf{n}$ bien grand. Quand l'installation est commode on peut refaire une mesure neuf fois, cela réduit l'erreur de la valeur $\mathbf{t h}$ a la valeur $\frac{\Delta h}{3}$. Il semble que ce soit la limite extrême de la pratique; par exemple, si on voulait attcindre l'exactitude marquée par $\frac{\Delta h}{4}$ il y faudrait seize opérations. On risquerait, par suite de la latigue inhérente au procédé, d'introduire dans la mesure des crreurs systématiques croissantes et même des fautes. On vérifierait une fois de plus que le mieux est l'ennemi du bien.

Si l'installation n'est pas très commode on derra s'estimer heureux de ramener $\Delta \mathbf{h} \grave{a} \frac{\Delta \mathbf{h}}{2}$ au moyen de quatre opé. rations.

Quant aux erreurs systématiques la répétition des mesures ne peut les compenser et il faut tâcher de les éliminer a priori et en bloc, ce qui est parfois délicat.

Done, quels que soient les efforts de l'ingénieur, une incertitude assez grande planera toujours sur le résultat de son jaugeage. C'est à lui d'aviser pour la délimiter et la réduire de son mieux.

\section{Jangeage dans des capacités définies; applications aux petits débits.}

Eı présence des difficultés que l'exposé précédent nous montre comme inhérentes aux procédés de jaugeage oú l'évaluation directe du temps est éliminée, on est amené à se demander s'il est réellement expédient de ne faire aucune mesure de durée, et s'il n'y aurait pas avantage à compter le temps qu'un courant donné d'eau met à remplir une capacité déterminée. On entend souvent préconiser cette méthode qui a le mérite d'être d'une conception simple; mais la simplicité ne doit pas s'acheter au prix de l'exacti. tude et, pour faire réussir ces procédés, il faut les cmployer avec de grandes précautions.

Ceux qui en douteraient n'ont qu'à se reporter aux ouvrages de Poncelet, Lebros et Boileau. Ils seront vite édifiés par le récit des soins et des précautions de toutes sortes que ces habiles et consciencieux observateurs ont dû prendre.

Cependant, nous croyons que c'est dans cette voie qu'i] faut chercher la solution du jaugeage des petits débits; les pages suivantes justifieront cette opinion, encore qu'on ne semble pas être dès mainterant en possession de procédés: irréprochalles.

Tout d'abord, il est bien évident que ce serait une utopie 
que de vouloir enfermer un débit, même moyen, pendant un temps, fût-il très court, dans une capacité fermée.

Pour les gros cours d'eau, nous n'aurons done que les jaugeages par limnimètres dont il a été question plus haut; quant aux courants de plus faible débit, lorsqu'il ne sera pas possible d'évaluer les charges avec une approximation moindre que I millimètre, il ne restera plus qu'à cuber, avec un soin méticuleux, une capacité assez grande pour que le temps nécessaire à son remplissage soit considérable en comparaison de l'importance des erreurs qu'on peut commettre dans la lecture des chronomètres.

$\mathrm{Si}$, par exemple, l'ingénieur ne peut apprécier que la seconde de temps, l'erreur sur la durée sera $\frac{\mathbf{1}}{\mathbf{3 6 0 0}}$ si le remplissage dure une heure, $\frac{1}{\mathbf{7 2 0 0}}$ s'il dure deux heures, etc.

L'avantage de la prolongation de la durée de l'épreuve est évident.

Quant à l'influence de l'exactitude dans le cubage, nous pouvons aisément nous en faire idće.

Supposons, pour fixer les idées, que nous ayions un bassin parallélipipédique rectangle exactement dressé et que $\mathbf{a}, \mathbf{b}, \mathbf{c}$, soient les longueurs de ses arêtes, de l'expression de son volume:

$$
\mathbf{V}=\mathbf{a} \mathbf{b} \mathbf{c}
$$

nous tirons :

$$
\lrcorner \mathbf{V}=\mathbf{a b} .\lrcorner \mathbf{c}+\mathbf{a c}\lrcorner \mathbf{b}+\mathbf{b a} .\lrcorner \mathbf{a}
$$

et :

$$
\frac{\Delta \mathbf{v}}{\mathbf{v}}=\frac{\Delta \mathbf{a}}{\mathbf{a}}+\frac{\Delta \mathbf{b}}{\mathbf{b}}+\frac{\Delta \mathbf{c}}{\mathbf{c}}
$$

Dans l'état actuel de l'outillage des mesures industrielles il n'est pas déraisonnable d'admettre qu'on aura les longueurs des arêtes du parallélipipède à $\frac{1}{\mathbf{5 0 0 0}}$ près.

Il en résulte :

$$
\frac{\Delta V}{V}=\frac{3}{5000}=\frac{6}{10^{4}}
$$

Ce qui peut, à bon droit, passer pour une très belle approximation.

Comme, d'autre part, le débit $\mathbf{Q}$ est, la durée étant marquée par $t, \quad \quad \quad Q=\frac{\mathbf{V}}{\mathbf{t}}$

$$
\begin{array}{ll}
\text { nous arons: } & \Delta \boldsymbol{Q}=\frac{\mathrm{t} \cdot \Delta \mathrm{V}-\mathrm{V} \Delta \mathrm{t}}{\mathrm{t}^{2}} \\
\text { et } & \frac{\Delta Q}{\mathbf{Q}}=\frac{\Delta \mathrm{V}}{\mathrm{V}}-\frac{\Delta \mathrm{t}}{\mathrm{t}}
\end{array}
$$

Si nous avons $\frac{\Delta t}{t}=+\frac{1}{3600}$ par exemple, il vient:

$$
\frac{\Delta \mathbf{Q}}{\mathbf{Q}}=\frac{393}{10^{\prime 3}}, \text { sensiblement } \frac{4}{10^{4}}
$$

C'est également une très belle approximation. Elle est même plus serrée, toutes relations gardées, que celle de la mesure du volume. Mais c'est là un fait accidentel. Si $\mathbf{d t}$ s'était produite en sens inverse, on aurait $\frac{\Delta t}{\mathbf{t}}=-\frac{\mathbf{1}}{\mathbf{3 6 0 0}}$ et il viendrait :

$$
\frac{\Delta \mathbf{Q}}{\mathbf{Q}}=\frac{\mathbf{8}}{\mathbf{1 0}} \text {, au minimum. }
$$

Au surplus, cette équation (18) mérite de nous arrêter quelque peu pour faire justice des interprétations paradoxales auxquelles elle pourrait donner lieu, faute de suffisante réflexion.

Supposons choisi, ne varietur, le volume $\mathbf{V}$ de la jauge, $\frac{\Delta \mathbf{V}}{\mathbf{V}}$ a alors une valeur fixe. $\Delta \mathbf{t}$ n'a pas forcément une valeur constante et, de plus, l'appréciation de la durée pouvant se faire tantôt par excès, tantôt par défaut, on ne peut pas assigner, à priori, un signe invariable à $\Delta \mathbf{t}$. Son signe sera donc tantôt celui de $\mathbf{J} \mathbf{V}$, tantôt le signe contraire. Imaginons que, par répartition opportune des termes dans les membres de droite ou de gauche de l'équation (i 8) nous fassions en sorte que $-\frac{\Delta t}{t}$ soit soustractif quand $\Delta t$ et $\Delta V$ sont de même sens et additif dans le cas contraire, nous aurons alors :

in Si $\lrcorner \mathbf{V}$ et $\boldsymbol{J t}$ sont de sens contraires :

$$
\frac{\Delta \mathbf{Q}}{\mathbf{Q}}-\frac{\Delta \mathbf{V}}{\mathbf{V}}+\frac{\Delta t}{\mathbf{t}}
$$

$\frac{\Delta \mathbf{Q}}{\mathbf{Q}}$ sera d'autant plus petit que $\mathbf{t}$ sera plus grand, et inversement.

$2^{\circ} \mathrm{Si} \Delta \mathrm{V}$ et $\Delta t$ sont de même sens, il vient :

$$
\frac{\Delta Q}{Q}=\frac{\Delta V}{V}-\frac{\Delta t}{t}
$$

plus $\mathbf{t}$ scra grand plus la valeur $\frac{\Delta \mathbf{Q}}{\mathbf{Q}}$ se rapprochera de celle de $\frac{\Delta \mathbf{V}}{\mathbf{V}}$ qui apparaît comme une limite supérieure.

$$
\text { Si } \mathbf{t}=\mathbf{V} \cdot \frac{\Delta \mathbf{V}}{\Delta \mathbf{t}} \text { l'erreur relative } \frac{\Delta \mathbf{Q}}{\mathbf{Q}} \text { est nulle. }
$$

Il serait très séduisant de réaliser le cas, mais on n'est jamais averti de l'avoir réalisé avec certitude, puisque l'on n'est maître ni du sens, ni de la valeur de $\Delta t$.

Si t est petit, $\frac{\Delta \mathbf{t}}{\mathbf{t}}$ peut, en valeur absolue, surpasser $\frac{\Delta \mathbf{V}}{\mathbf{V}}$ et alors $\frac{\Delta \mathbf{Q}}{\mathbf{Q}}$ peut croître de manière démesurée.

Nous voyons donc que, sauf pour quelques valeurs spéciales, impossibles à maîtriser en pratique, il y a tout intérêt à ce que $\mathbf{t}$ soit grand, donc à ce que $\mathbf{V}$ le soit aussi. Ce serait donc verser dans un paradoxe que de s'autoriser de la forme de l'équation (18) pour soutenir que, plus $\mathbf{t}$ sera petit, plus $\frac{\Delta \boldsymbol{Q}}{\boldsymbol{Q}}$ sera obtenu avec exactitude. C'est lc contraire qui est la vérité générale et pratique.

Pour les cas où $\frac{\Delta \mathbf{Q}}{\mathbf{Q}}$ augmente avec l'augmentation de la valeur de $\mathbf{t}$, il est tout indiqué de taire $\mathbf{V}$ très grand pour que $\frac{\Delta \mathbf{V}}{\mathbf{V}}$ limite vers laquelle tend alors $\frac{\Delta \mathbf{Q}}{\mathbf{Q}}$, soit aussi petit que possible. 
En résumé, il faut donc, toutes choses égales d'ailleurs, jauger dans les capacités les plus grandes que l'on pourra. Mais alors c'est sur les dimensions véritables que porteront les augmentations. Si les bassins de jauge sont en terre vierge leur régularité sera médiocre et ils s'affouilleront très vite; avec de la maçonnerie on pourra avoir plus de régularité, mais au prix de soins méticuleux et de frais considérables ( $\mathrm{t}$ ). Cette solution ne semble pas convenir à la pratique industrielle et c'est alors en métal que les bassins pourront être faits avec le plus d'avantage. Cela dépasse les bornes de l'improvisation, parfois même celle de la pratique, parce que c'est surtout pour les hautes chutes que les petits débits sont intéressants. Or, s'il faut commencer les études par le transport à de hautes altitudes d'un matériel de mesure encombrant et lourd, beaucoup trouveront la dépense trop onéreuse.

Avec toute autre forme du bassin de jauge, les inconvénients que nous venons d'analyser seraient encore plus marqués, tant à cause de la difficulté d'exécution de l'objet que par suite de la difficulté d'en mesurer exactement les dimensions.

Donc: impraticable, même pour les débits moyens ou qu'ils se trouvent : difficile pour les petits débits des torrents élevés, telle nous apparaît la méthode prise dans l'ensemble des cas. Cela ne veut pas dire que, dans des circonstances propices, mais rares (ce sur quoi nous voulons surtout insister), elle ne puisse donner de très bons résultats, mais il ne faut l'employer qu'à bon escient.

Imaginons, par exemple, qu'on ait pu installer un récipient de $1^{m^{3}}$ en métal et que le débit ait été tel que ce mètre cube ait été rempli exactement en $16^{\text {min. }} \mathbf{4 0}$ sec. $=1000 \mathrm{sec}$. $\mathrm{Si}$ on a apprécié la durée avec une montre à secondes,

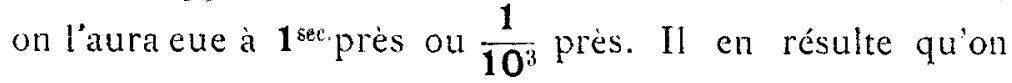
aura :

$$
\frac{\Delta \mathbf{Q}}{\mathbf{Q}}=\frac{6}{10^{4}}-\frac{1}{10^{3}}=-\frac{4}{10^{4}}
$$

soit s mètre cube mesuré à $2 / 5$ de litre près, ce qui est assurément fort bien. Mais, si le courant avait été plus rapide et qu'au lieu de remplir le mètre cube en rono secondes on l'eût "rempli en 100 , l'approximation eùt été :

$$
\frac{\Delta Q}{Q}=\frac{6}{10^{1}}-\frac{1}{10^{2}}=-\frac{94}{10^{4}}
$$

ou 9 litres $\uparrow_{4}$, et ainsi de suite.

On voit donc qu'il $y$ a matiere à discussion avant d'employer ce procédé, pourtant très tentant (2).

(I) Voir un aperçu des dépenses que peut nécéssiter une telle cuvre dans le Bulletin de la Societé de Statistique de Grenoble, de 1899 . Règlements d'eau entre les bassins de Domène et de Lancey. Communication de M. R. de la Brosse.

(2) L'équation (13) nous montre que si :

$$
\Delta \mathbf{c}=\Delta \mathbf{b}-\Delta \mathbf{a}
$$

ce qui arrive souvent dans la pratique, ce sera l'erreur commise sur le plus petit côté (admettons que ce soit e) qui aura la plus grande intluence sur la valeur absolue de l'erreur $\Delta \mathrm{V}$ du résultat, parce que cette erreur partielle est celle qui a le plus grand multiplicateur ab. C'est là une particularité connue des marchands qui, lorsqu'ils débitent leurs marchandises à tant la superticie ou à tant le volume, font volontiers des concessions à l'acheteur sur les grandes dimensions et non sur les petites, ayant ainsi l'air d'être très désintéressés. Or, c'est une pure apparence, car la différence entre les diverses valeurs que peut prendre $\Delta V$ est un bénéfice pur eux et ils le rendent ainsi maximum.

C'est une remarque qu'il faut aussi mettre a profit dans la cubature des bassins de jauge, afin de diriger raisonnablement les mesures.

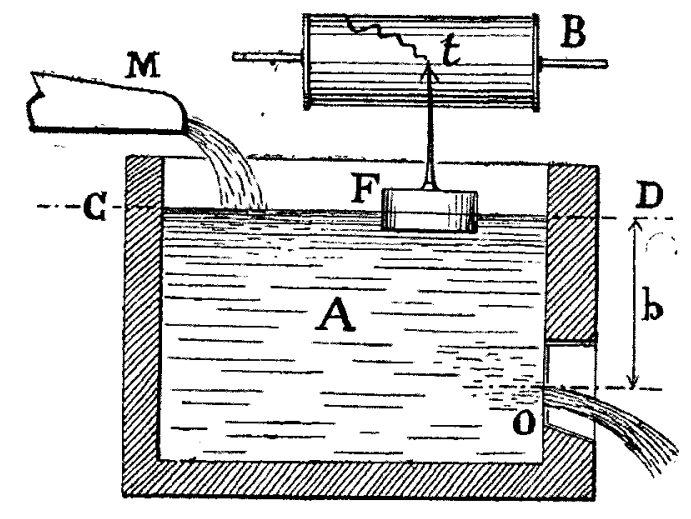

Ia question du jaugeage des petits debits est si importante que l'on ne sétonnera pas de nous voir insister sur les procédés qui semblent devoir en permettre une mesure facile et rigoureuse, si cela se peut. Le journal La Nature, si notre mémoire est bonne, a donné, dans ces dernières années, l'indication d'un dispositif qui pourrait aisément être mis à profit en pareil cas. Nous en donnons l'indication schématique ci-contre.

A est un récipient de torme géométrique quelconque. On y fait couler l'eau du courant à jauger jusqu'à ce qu'elle monte au niveau convenable GD. A ce moment, on ouvre l'orifice $\boldsymbol{O}$, percé en minces parois, d'une quantité suffisante pour que son débit soit égal à lapport fait par la rigole $\mathbf{M}$. - CD va rester sensiblement constant. Si on appuie sur le niveau un flotteur F, muni d'un style portecrayon $\mathbf{t}$,et qu'on fasse tourner un tambour à inscription $\mathbf{B}$, avec une vitesse déterminée (par exemple un tour à l'heure) devant le style, les fluctuations du niveau CD s'enregistreiont à leur heure sur la bande de papier du tambour. On aura ainsi les diverses valeurs de la charge $\mathbf{h}$ sur l'orifice d'écoulement à tous instants, par suite le débit exact (á l'exactitude près des formules de Poncelet et Lesbrosi par cet orifice, donc le débit du courant qui Jui est égal. On aura le débit moven à telle époque, etc., etc.

Si on munissait le style $t$, d'un dispositif amplificateur, on mettrait encore plus de précision dans les lectures ; entin, en contrariant, par un système de cloisons en chicane, les remous formés par la chute de l'ear de $\mathbf{M}$ en $\mathbf{A}$, on supprimerait tous les tremblements parasites de la courbe $t$.

Ce dispositif est donc très séduisant, surtout par sa facilité d'installation et d'emploi. Son inconvénient nous semble résider dans les erreurs systématiques et accidentelles que l'appareil introduit fatalement dans les mesures, car on voit qu'il n'élimine aucune des causes derreurs que nous avons analysées sur la durée, la charge, l'emploi des formules: il les assemble même toutes ensemble. Il est vrai qu'il peut permettre d'en apprécier quelques-unes, comme le temps, avec quelque rigueur. Il n'est, sans doute, pas impossible non plus de tarer l'appareil: il le fiut même faire de son mieux, mais c'est là une complication. Enfin, bien qu'il ne soit, sans doute, ni très difficile, ni très onéreux de se procurer séparément les diverses partie de l'instrument (horlogerie, flotteur, récipient, cylindre enregistreur, atc.) et de les assembler, le prix doit cependant entrer aussi en ligne de compte.

\section{Commandant Audrirand, Ingénieur,}

(A suivre) 